\title{
Excited-state effects in nucleon structure on the lattice using hybrid interpolators
}

\author{
Jeremy R. Green $\odot,{ }^{1, *}$ Michael Engelhardt, ${ }^{2}$ Nesreen Hasan $\odot,{ }^{3,4}$ Stefan Krieg $\oplus^{3,4}$ Stefan Meinel®, ${ }^{5,6}$ \\ John W. Negele, ${ }^{7}$ Andrew V. Pochinsky, ${ }^{7}$ and Sergey N. Syritsyn ${ }^{6,8}$ \\ ${ }^{1}$ NIC, Deutsches Elektronen-Synchroton, 15738 Zeuthen, Germany \\ ${ }^{2}$ Department of Physics, New Mexico State University, Las Cruces, New Mexico 88003-8001, USA \\ ${ }^{3}$ Bergische Universität Wuppertal, 42119 Wuppertal, Germany \\ ${ }^{4}$ IAS, Jülich Supercomputing Centre, Forschungszentrum Jülich, 52425 Jülich, Germany \\ ${ }^{5}$ Department of Physics, University of Arizona, Tucson, Arizona 85721, USA \\ ${ }^{6}$ RIKEN BNL Research Center, Brookhaven National Laboratory, Upton, New York 11973, USA \\ ${ }^{7}$ Center for Theoretical Physics, Massachusetts Institute of Technology, Cambridge, \\ Massachusetts 02139, USA \\ ${ }^{8}$ Department of Physics and Astronomy, Stony Brook University, Stony Brook, New York 11794, USA
}

(Received 5 August 2019; published 24 October 2019)

\begin{abstract}
It would be very useful to find a way of reducing excited-state effects in lattice QCD calculations of nucleon structure that has a low computational cost. We explore the use of hybrid interpolators, which contain a nontrivial gluonic excitation, in a variational basis together with the standard interpolator with tuned smearing width. Using the clover discretization of the field strength tensor, a calculation using a fixed linear combination of standard and hybrid interpolators can be done using the same number of quark propagators as a standard calculation, making this a cost-effective option. We find that such an interpolator, optimized by solving a generalized eigenvalue problem, reduces excited-state contributions in two-point correlators. However, the effect in three-point correlators, which are needed for computing nucleon matrix elements, is mixed: for some matrix elements such as the tensor charge, excited-state effects are suppressed, whereas for others such as the axial charge, they are enhanced. The results illustrate that the variational method is not guaranteed to reduce the net contribution from excited states except in its asymptotic regime, and suggest that it may be important to use a large basis of interpolators capable of isolating all of the relevant low-lying states.
\end{abstract}

DOI: 10.1103/PhysRevD.100.074510

\section{INTRODUCTION}

One of the most challenging sources of systematic uncertainty faced by lattice QCD calculations of nucleon structure is excited-state contamination: the failure to isolate the ground-state nucleon from the tower of higher-energy states to which the interpolating operator can couple. Although the unwanted excited states can be exponentially suppressed by Euclidean time evolution, this is hindered by an exponentially decaying signal-to-noise ratio [1] that makes it impractical to evolve long enough in Euclidean time.

The variational method [2-4] provides a way of improving the interpolating operator such that the lowest-lying

\footnotetext{
*jeremy.green@desy.de
}

Published by the American Physical Society under the terms of the Creative Commons Attribution 4.0 International license. Further distribution of this work must maintain attribution to the author(s) and the published article's title, journal citation, and DOI. Funded by SCOAP. excited states can be systematically removed. Variational approaches have been used to study nucleon structure in Refs. [5-9], which used bases of interpolators with different smearing widths and different site-local spin structures; Refs. [10-12], which used bases with the standard interpolator evolved by different Euclidean time intervals, i.e., the generalized pencil-of-function method $[13,14]$; and Ref. [15], which used the distillation method to enable the use of interpolators with a variety of local structures including covariant derivatives. In these cases, the variational setup was more computationally expensive than a standard calculation because of the need for additional quark propagators with different smeared sources or (for time-evolved operators) additional source-sink separations. ${ }^{1}$

In this paper, we present a study of a variational setup that requires the same number of quark propagators as a standard calculation, for a fixed choice of optimized

\footnotetext{
${ }^{1}$ The comparison is more difficult when using the distillation method, which uses timeslice sources rather than the point sources used in standard calculations.
} 
interpolator. This is accomplished by supplementing the standard interpolator with hybrid ones [16] that contain a gluonic excitation. ${ }^{2}$ In Ref. [16], it was found that the latter have the next-largest overlaps onto the ground state, after the standard interpolator, but overlap much more strongly than the standard interpolator with certain high-lying excited states. The use of hybrid interpolators presents the possibility of an improvement over the standard approach at low computational cost.

It should be stressed that this study, along with all previous ones in the nucleon sector, ${ }^{3}$ uses only local interpolating operators, which are poor at isolating multiparticle states. In practice, the true spectrum that includes $N \pi$ - and $N \pi \pi$-like states is not identified, meaning that the calculation is not in a regime where the variational method has been proven to improve the isolation of the ground state. Therefore, the question of whether one interpolator is an improvement over another is an empirical one, to be decided by examining excited-state contributions in estimators for a variety of observables.

This paper is organized as follows. Section II discusses our lattice setup, the basis of interpolating operators, and two tuning runs. Results for two-point correlators are discussed in Sec. III A, forward matrix elements are shown in Sec. III B, and form factors are presented in Sec. III C. Our conclusions are given in Sec. IV.

\section{LATTICE SETUP}

As this is an exploratory calculation, we use a single lattice ensemble with a coarse lattice spacing at a heavier-thanphysical pion mass and a relatively small box size; its parameters are summarized in Table I. This has $2+1$ flavors of tree-level improved Wilson-clover quarks coupled to the gauge links via two levels of HEX smearing [18].

Aside from the interpolating operator, the methodology used here for computing nucleon matrix elements and form factors is unchanged from previous work such as Ref. [11]; the reader is referred to that earlier work for details. Our focus is on seeing whether excited-state contamination can be reduced, and therefore we use three relatively short source-sink separations, $T$, ranging from 0.70 to $1.16 \mathrm{fm}$. We use two methods for determining matrix elements: the ratio method-for which the asymptotically leading excited-state contributions decay as $e^{-\Delta E T / 2}$, where $\Delta E$ is the energy gap to the lowest excited state-and the summation method, for which they decay as $T e^{-\Delta E T}$.

Given a set of $N$ interpolating operators $\left\{\chi_{i}\right\}$, one would like to find a linear combination $\chi=v_{i} \chi_{i}$ that has a reduced coupling to excited states. The standard approach is to compute a matrix of two-point correlators,

\footnotetext{
${ }^{2}$ The resulting basis of interpolators is similar to the one called $\mathcal{B}_{3}$ in Ref. [15].

${ }^{3}$ Bilocal operators were used in Ref. [17], but that was a study of only spectroscopy and not structure.
}

$$
C_{i j}(t)=\left\langle\chi_{i}(t) \chi_{j}^{\dagger}(0)\right\rangle
$$

and then solve a generalized eigenvalue problem (GEVP)

$$
C\left(t_{2}\right) v=\lambda C\left(t_{1}\right) v
$$

for some choice of $\left(t_{1}, t_{2}\right)$. It has been shown [4] that by suitably increasing $t_{1}$ and $t_{2}$ to remove contributions from higher excited states in the determination of $v$, one can define improved estimators for the ground-state energy and matrix elements, for which the leading excited-state effect depends on the energy gap to state $N+1$ rather than the second (i.e., first excited) state. However, it is known that for light pion masses and large volumes, the number of low-lying excited states with the quantum numbers of the nucleon is large due to the presence of multiparticle $(N \pi$, $N \pi \pi$, etc.) states [19-26]. Removing the effects of these states would require that the basis include at least one operator for each state. In addition, it has been found in meson spectroscopy calculations that nonlocal multiparticle interpolators must be included in order to correctly identify the multiparticle spectrum: see, e.g., Ref. [27]. For nucleons, the need for nonlocal operators is also supported by chiral perturbation theory, which predicts at leading order that the ratio of couplings for single nucleon and nucleon-pion states is the same for all local operators [21]. This defeats the diagonalization procedure of Eq. (2) such that $N \pi$ states cannot be removed.

The challenge of systematically removing all contributions from the lowest-lying excited states will be left to future work. Instead, we hope to find an improved local operator that can be used in existing software with minimal modifications and with little additional computational cost. Our standard operator is

$$
\chi_{1}=\epsilon_{a b c}\left(\tilde{u}_{a}^{T} C \gamma_{5} P_{+} \tilde{d}_{b}\right) \tilde{u}_{c},
$$

where $P_{+}=\left(1+\gamma_{4}\right) / 2$ is a positive parity (nonrelativistic) projector and $\tilde{q}$ is a smeared quark field. When used in a two-point or three-point correlator with a polarization matrix that includes a factor of $P_{+}$, the projector is applied to all three quarks. This allows for computational cost savings in the quark propagators used for constructing correlators: only half of the propagator solves are required [28]. Of the three possible site-local nucleon operators, $\chi_{1}$ is the only one that can be constructed using positiveparity-projected quark fields (see e.g., Appendixes B and C of Ref. [29]).

We also consider hybrid operators, introduced in Ref. [16], which include an insertion of the chromomagnetic field $B_{i}=-\frac{1}{2} \epsilon_{i j k} F_{j k}$ and are interpreted as having a nontrivial gluonic excitation. Using the clover discretization of $F_{\mu \nu}$ [30], no additional quark propagators are needed for constructing two-point correlators using hybrid operators. Two different nucleon operators exist that use positive-parity-projected quark fields: 
TABLE I. Parameters of the ensemble and measurements used in this work. The lattice spacing is taken from Ref. [18], where it is set using the mass of the $\Omega$ baryon at the physical point. $N_{\text {conf }}$ refers to the number of gauge configurations analyzed and $N_{\text {meas }}=$ $2 \times N_{\text {conf }} \times N_{\text {src }}$ is the number of measurements performed. The factor of 2 in $N_{\text {meas }}$ accounts for the use of forward- and backwardpropagating states.

\begin{tabular}{lccccccccccc}
\hline \hline Size & $\beta$ & $a m_{u d}$ & $a m_{s}$ & $a[\mathrm{fm}]$ & $a m_{\pi}$ & $m_{\pi}[\mathrm{MeV}]$ & $m_{\pi} L$ & $T / a$ & $N_{\text {conf }}$ & $N_{\text {src }}$ & $N_{\text {meas }}$ \\
\hline $24^{3} \times 48$ & 3.31 & -0.09530 & -0.04 & $0.1163(4)$ & $0.1499(7)$ & $254(1)$ & 3.6 & $\{6,8,10\}$ & 600 & 48 & 57600 \\
\hline \hline
\end{tabular}

$$
\begin{aligned}
\chi_{2}= & \epsilon_{a b c}\left[\left(B_{i} \tilde{u}\right)_{a}^{T} C \gamma_{j} P_{+} \tilde{d}_{b}\right] \gamma_{i} \gamma_{j} \tilde{u}_{c} \\
& -\epsilon_{a b c}\left[\tilde{u}_{a}^{T} C \gamma_{j} P_{+}\left(B_{i} \tilde{d}\right)_{b}\right] \gamma_{i} \gamma_{j} \tilde{u}_{c}, \\
\chi_{3}= & \epsilon_{a b c}\left[\left(B_{i} \tilde{u}\right)_{a}^{T} C \gamma_{j} P_{+} \tilde{d}_{b}\right] P_{i j} \tilde{u}_{c} \\
& -\epsilon_{a b c}\left[\tilde{u}_{a}^{T} C \gamma_{j} P_{+}\left(B_{i} \tilde{d}\right)_{b}\right] P_{i j} \tilde{u}_{c},
\end{aligned}
$$

where $P_{i j}=\delta_{i j}-\frac{1}{3} \gamma_{i} \gamma_{j}$. These differ in the spin of the three quarks: for $\chi_{2}$ it is $\frac{1}{2}$ and for $\chi_{3}$ it is $\frac{3}{2}$. In both cases, this is combined with the chromomagnetic field to produce an overall spin of $\frac{1}{2}$.

Our production strategy begins with two tuning runs where only two-point correlators are computed. The first uses only $\chi_{1}$ and serves to select the quark smearing parameters. The second serves for determining the coefficients $v_{i}$ of an optimized operator $\chi_{\mathrm{opt}}=v_{i}^{*} \chi_{i}$. These are followed by a production run with higher statistics in which both two-point and three-point correlators are computed. The three-point correlators are computed using the standard operator $\chi_{1}$ and the linear combination $\chi_{\mathrm{opt}}$, in both cases keeping the same operator at the source and the sink. For three-point correlators, using $\chi_{\mathrm{opt}}$ at the source and the sink requires a different sequential propagator than for $\chi_{1}$, but the total number of propagators needed in each case is the same. We chose not to compute all nine combinations of source and sink interpolators in three-point correlators because this would require nine times as many sequential propagators as a standard calculation.

\section{A. Tuning of quark smearing}

We use Wuppertal smearing [31], $\tilde{q} \propto(1+\alpha H)^{N} q$, where $H$ is the nearest-neighbor gauge-covariant hopping matrix constructed using the same smeared links used in the fermion action. The parameter $\alpha$ is fixed to 3.0 , and $N$ is varied to produce different smearing widths. The smearing radius is determined by taking a color field $\varphi(\vec{x})$ with support only at the origin and then defining a density from the squared norm of the smeared field: $\rho(\vec{x})=|\tilde{\varphi}(\vec{x})|^{2}$. Finally, we take the root-mean-squared radius:

$$
r^{2}=\frac{\sum_{\vec{x}}|\vec{x}|^{2} \rho(\vec{x})}{\sum_{\vec{x}} \rho(\vec{x})}
$$

For the tuning run, we used $N \in\{20,40,70,110,160\}$, which correspond roughly to $r / a \in\{3,4,5,6,7\}$.

Figure 1 shows the effective mass $a_{\mathrm{eff}}(t)=\log \frac{C(t)}{C(t+a)}$ for each smearing width. For $t=2 a$ and $3 a$, we can see that the minimum lies near $N=40$ and 70 . Based on this, we decided to use the same smearing parameter, $N=45$, that was used in a previous calculation $[11,32,33]$.

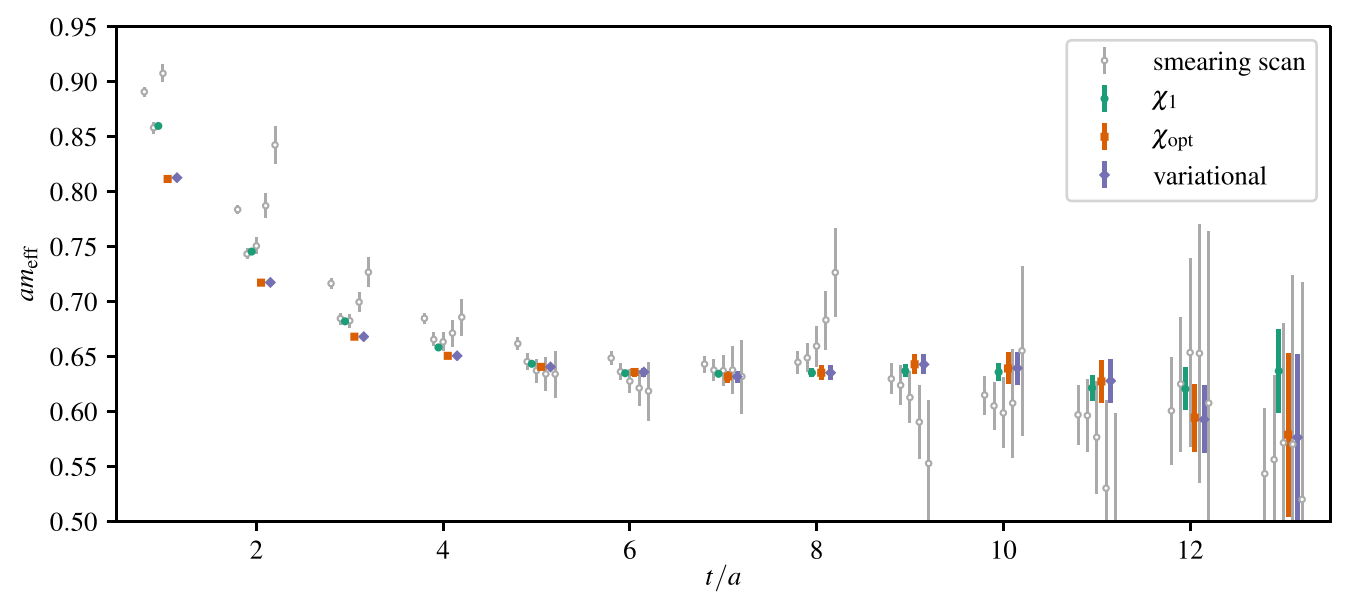

FIG. 1. Effective mass of the nucleon. The five open gray circles at each $t$ show results from the low-statistics tuning of the quark smearing: the number of smearing steps increases from left to right. Filled symbols are from the full-statistics run: the standard operator (green circles), $\chi_{\text {opt }}$ chosen based on the second tuning run (orange squares), and the variationally optimized operator based on the fullstatistics run (blue diamonds). 


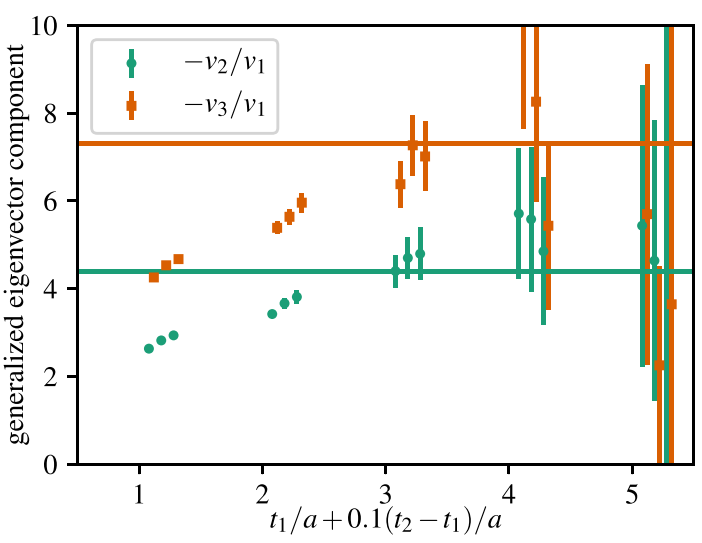

FIG. 2. Components of the generalized eigenvector $v_{i}$, normalized to $v_{1}$. The GEVP was solved for $t_{1} / a \in[1,5]$ and $\left(t_{2}-t_{1}\right) / a \in[1,3]$. The horizontal lines indicate the values used in the optimized operator $\chi_{\mathrm{opt}}=v_{i} \chi_{i}$, determined from the lowerstatistics tuning run.

\section{B. Tuning of variational operator}

The hybrid operators are constructed using a chromomagnetic field made from smeared gauge links. We take the smeared links to which the fermions couple in the action and then apply additional three-dimensional stout smearing $^{4}$ [34]: 20 steps with $\rho=0.1$. The traceless part of the clover definition of the field strength tensor is used.

For the second tuning run, we computed the full $3 \times 3$ matrix of two-point correlators. Solving the GEVP yielded the coefficients $v_{i}$ for $\chi_{\text {opt }}$; we did this at the largest available time separations before the noise became too large. For our choice of operators, the correlator matrix is real and thus $v_{i}$ are also real. Normalizing such that $v_{1}=1$, we selected $\chi_{\text {opt }}=\chi_{1}-4.4 \chi_{2}-7.3 \chi_{3}$. The determination of coefficients from the subsequent full-statistics run is shown in Fig. 2 for a range of $t_{1}$ and $t_{2}$ in the GEVP. Our selection based on the tuning run is consistent with the values determined at large times in the full run.

\section{RESULTS}

\section{A. Two-point correlators}

The full $3 \times 3$ matrix of two-point correlators was computed in the full-statistics run, allowing for a more detailed analysis. We begin by determining the excited energy levels using the variational method. Solving the GEVP at $\left(t_{1}, t_{2}\right)=(3 a, 5 a)$ yields an eigenvector $v_{n}$ for each state $n$. This allows us to define projected correlators $C_{n}(t)=v_{n}^{\dagger} C(t) v_{n}$ and then compute their effective energies; these are shown in Fig. 3. The two excited energies are nearly degenerate and lie in the range from 1 to $1.5 \mathrm{GeV}$ above the ground-state nucleon; this is similar to the hybrid states observed in Ref. [16]. However, we stress

\footnotetext{
${ }^{4}$ We have not studied the effect of varying this.
}

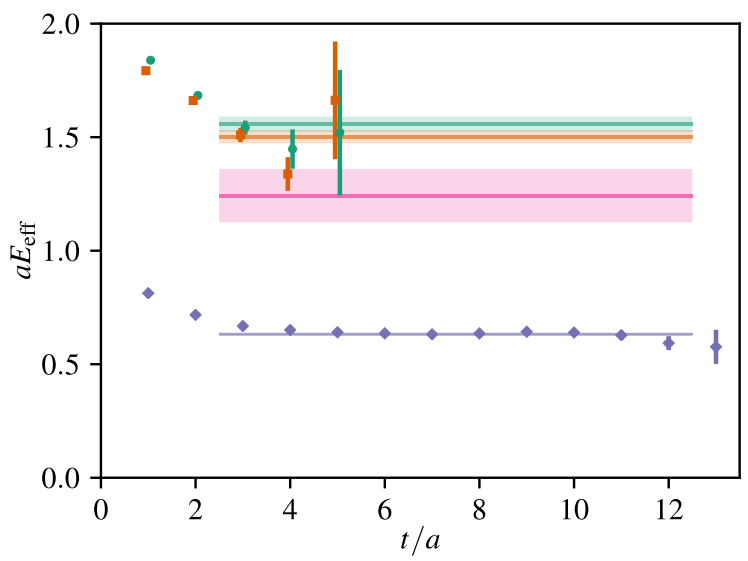

FIG. 3. Effective energies for the three GEVP-projected correlators at zero momentum (in order of increasing energy: blue diamonds, orange squares, and green circles) and energies from the four-state fit (horizontal bands). The colors of the bands correspond to the effective energy with which they are identified in Sec. III A; the additional state has a magenta band. The horizontal range of the bands indicates the time separations in the two-point correlator matrix that are fitted.

that this should not be considered a reliable determination of the spectrum, as many lower-lying excitations are expected and cannot be identified using our small basis of three operators.

The presence of residual excited-state effects in the effective mass (Fig. 1) suggests that more than three states are needed to describe the two-point correlators. We employ the fit model
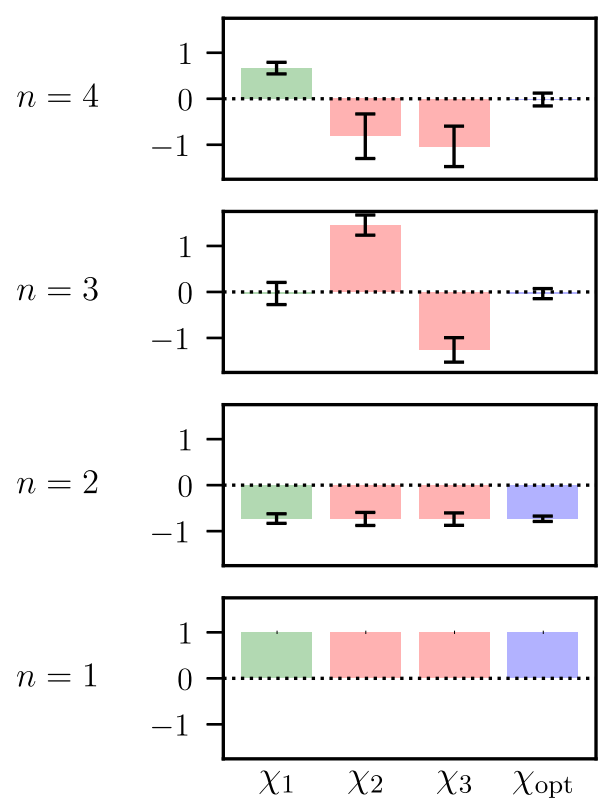

FIG. 4. Overlap factors normalized to the ground state, $Z_{i, n} / Z_{i, 1}$. These are shown for the three operators $\chi_{i}$ and for their linear combination $\chi_{\text {opt }}$. 

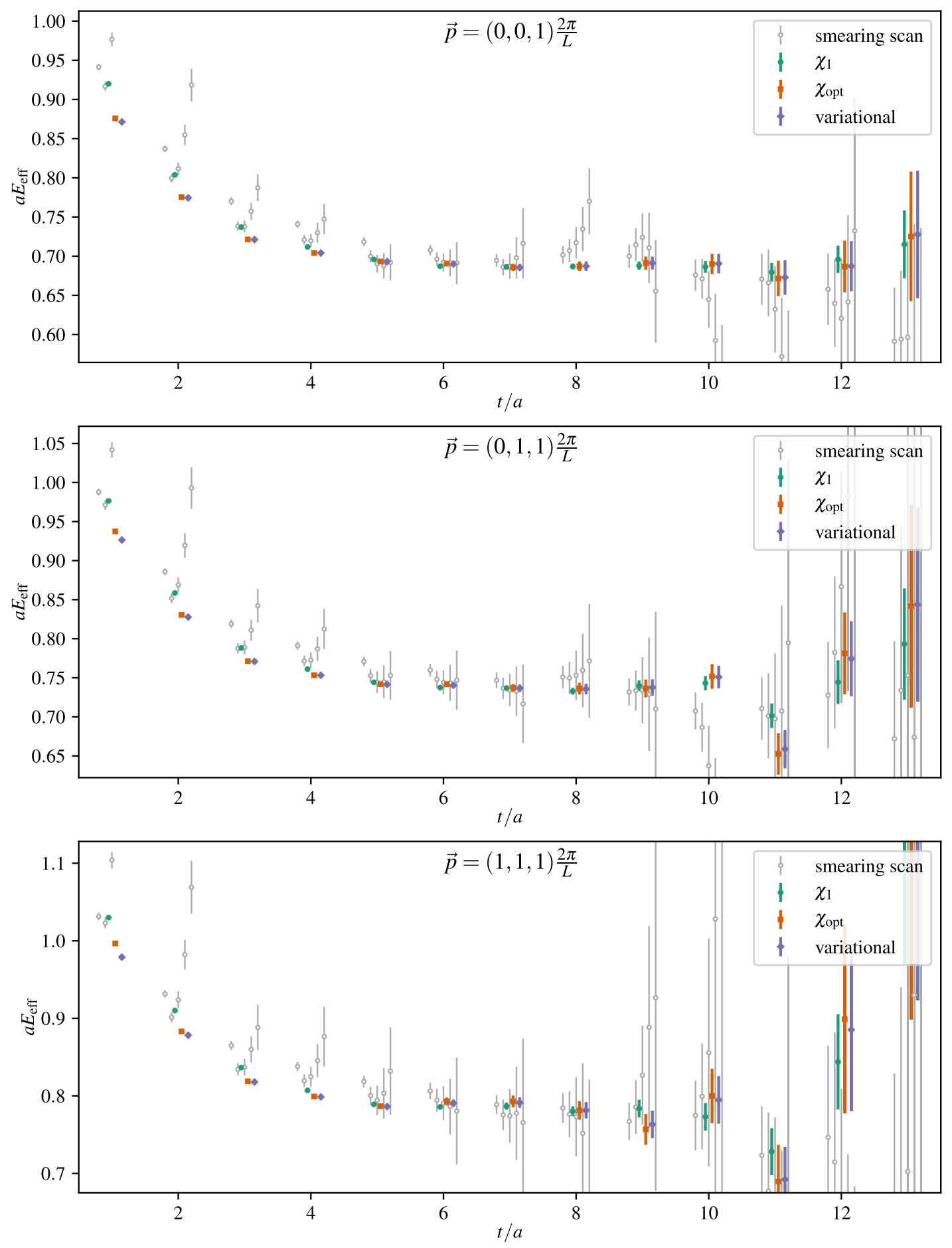

FIG. 5. Effective energies of the nucleon at nonzero momentum. See the caption of Fig. 1. Note that $\chi_{1}$ and $\chi_{\text {opt }}$ were tuned at zero momentum, whereas the full variational analysis is retuned at nonzero momentum.

$$
C_{i j}(t)=\sum_{n=1}^{N_{\text {states }}} Z_{i, n} Z_{j, n} e^{-E_{n} t},
$$

with the energies ordered $E_{1}<E_{2}<\cdots$. We obtained a good fit to the range $t / a \in[3,12]$ using $N_{\text {states }}=4$, which yielded $\chi^{2} /$ dof $=0.89(p=0.68)$. The four energy levels are shown in Fig. 3. States 3 and 4 are consistent with the effective energies from the GEVP-projected correlators for the two excited states with $t / a=3$. The additional energy level, state 2 , sits below the two excited states identified by the GEVP.

This identification of states between the GEVP and the four-state fit is also supported by Fig 4, which shows the overlap factors normalized to the ground state, 

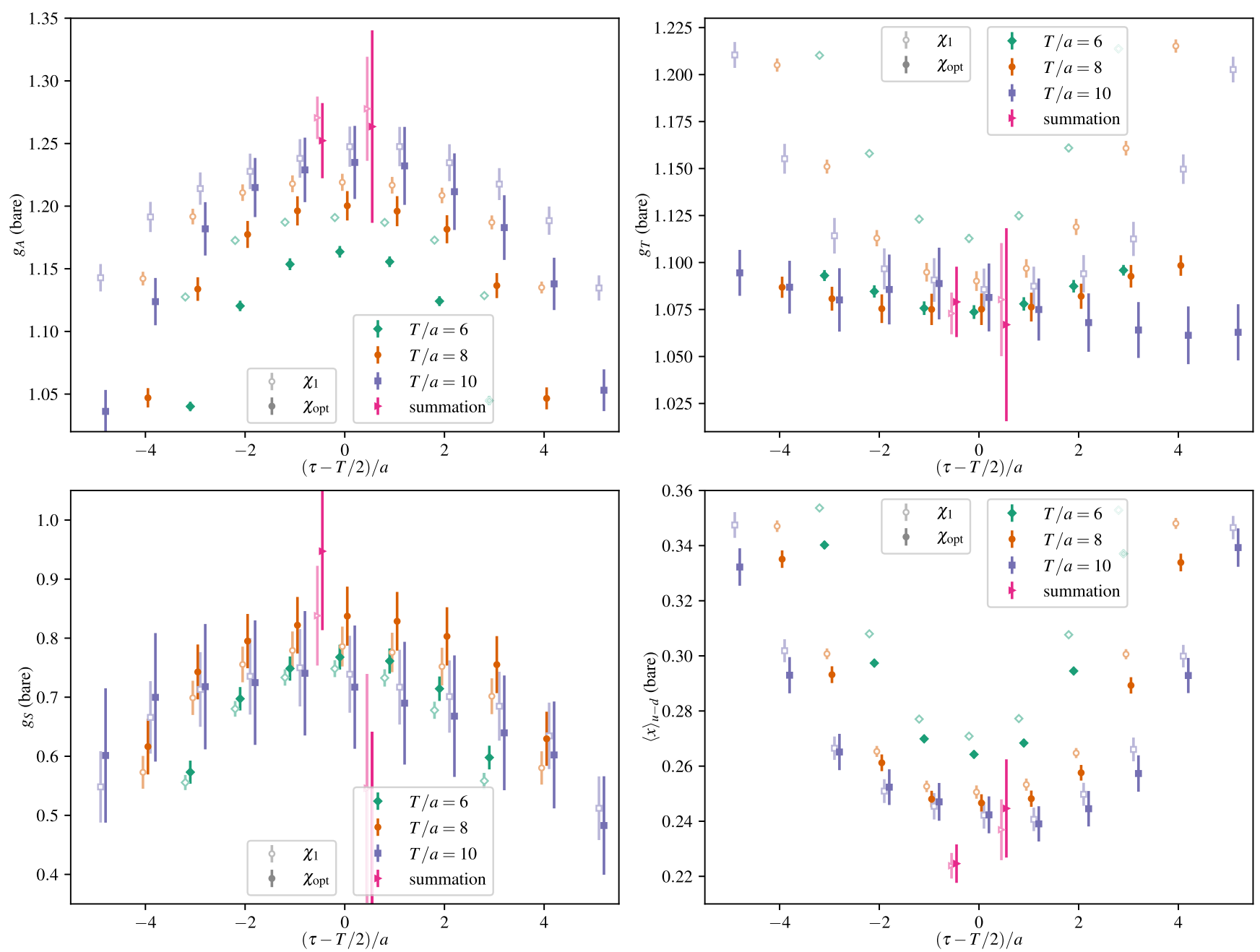

FIG. 6. Isovector axial, tensor, and scalar charges $\left(g_{A}, g_{T}\right.$, and $\left.g_{S}\right)$ and isovector momentum fraction $\langle x\rangle_{u-d}$ determined using the standard interpolator $\chi_{1}$ (open symbols) and the linear combination of standard and hybrid interpolators $\chi_{\text {opt }}$ (filled symbols). Ratiomethod data are shown for source-sink separations $T / a=6$ (green diamonds), 8 (orange circles), and 10 (blue squares), and are plotted versus the operator insertion time $\tau$, shifted by half the source-sink separation. Summation-method (magenta triangles) data are based on the discrete derivative of sums, $[S(T+2 a)-S(T)] /(2 a)$; the two points correspond to $T / a=6$ and 8 .

$Z_{i, n} / Z_{i, 1}$. It also shows the normalized overlap factors for the operator $\chi_{\mathrm{opt}}$, which are determined via $Z_{\mathrm{opt}, n}=v_{i} Z_{i, n}$. The fit results indicate that $\chi_{1}$ has a significant overlap with state 4 , which is eliminated in $\chi_{\text {opt }}$. The overlap of $\chi_{1}$ with state 3 is consistent with zero, and this is preserved in $\chi_{\text {opt }}$ despite the large overlaps of $\chi_{2}$ and $\chi_{3}$ with state 3 . The operators show no significant difference in the relative overlaps with states 1 and 2; because of this, the GEVP was insensitive to state 2 and was unable to eliminate the coupling of $\chi_{\text {opt }}$ to it.

Finally, we can compare effective energies produced using different operators. In addition to $\chi_{1}$ and $\chi_{\text {opt }}$, which were tuned at zero momentum using reduced statistics, we also perform a new variational analysis based on the fullstatistics run. In Fig. 1, there is no significant difference between $\chi_{\text {opt }}$ and the new variational operator. Both of them have smaller effective masses than $\chi_{1}$ at early times, indicating a significant reduction in excited-state contributions. However, they also suffer from increased statistical uncertainty. Figure 5 shows the same comparison at nonzero momentum. In all cases, $\chi_{\text {opt }}$ shows smaller excitedstate effects than $\chi_{1}$. At the smallest values of $t$, the new variational operator also shows an improvement over $\chi_{\text {opt }}$, and this effect grows with momentum. This is not surprising, as the new variational operator is tuned for each momentum, whereas $\chi_{\text {opt }}$ was chosen at $\vec{p}=0$.

\section{B. Forward matrix elements}

We have only computed two combinations of source and sink interpolators in three-point correlators: those with the same interpolator at the source and the sink, which is chosen to be either $\chi_{1}$ or $\chi_{\text {opt }}$. For comparing these two setups, we start by considering observables computed from isovector matrix elements at zero momentum: the axial, 

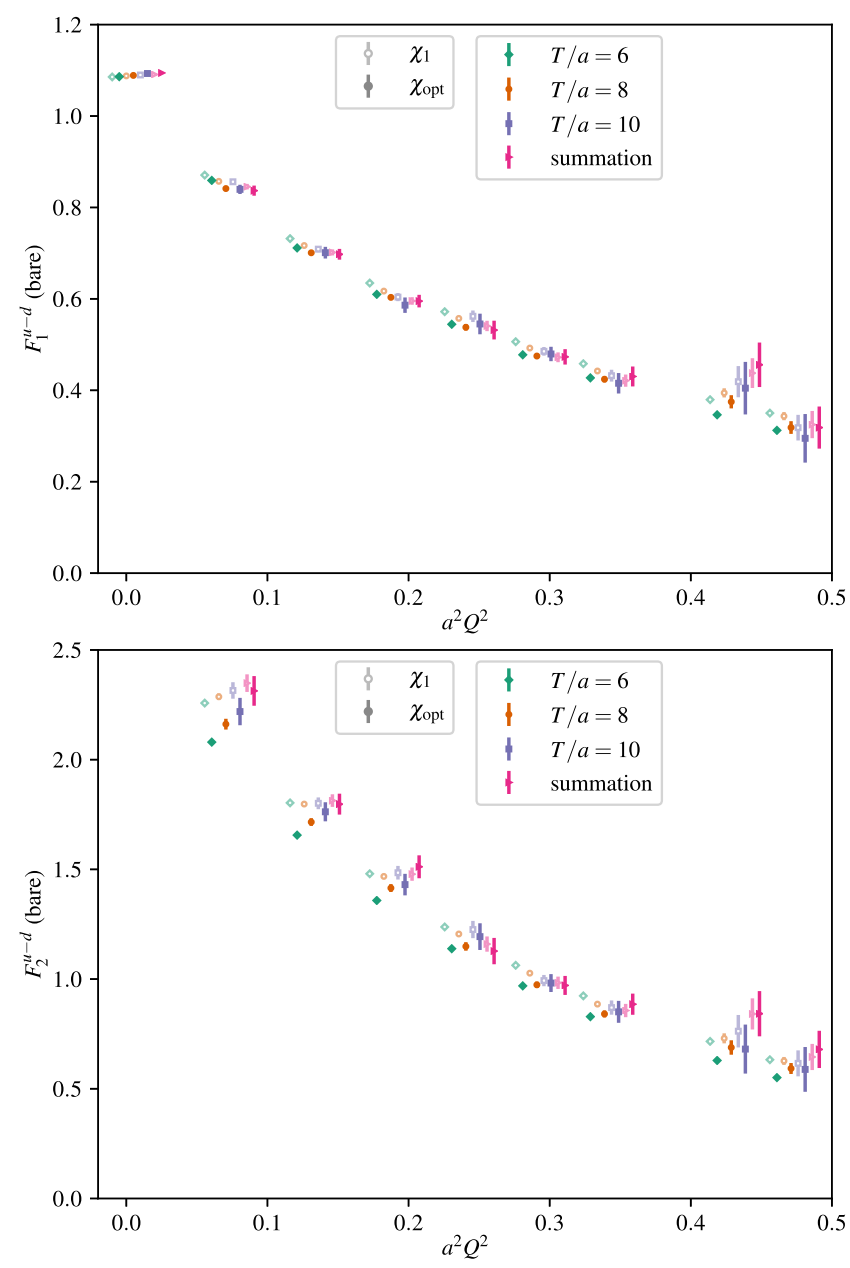

FIG. 7. Isovector Dirac and Pauli form factors, $F_{1}$ and $F_{2}$, determined using the standard interpolator $\chi_{1}$ (open symbols) and the linear combination of standard and hybrid interpolators $\chi_{\text {opt }}$ (filled symbols). Ratio-method data are shown for source-sink separations $T / a=6$ (green diamonds), 8 (orange circles), and 10 (blue squares). Summation-method (magenta triangles) data are based on fitting a line to the three sums.

tensor, and scalar charges $\left(g_{A}, g_{T}\right.$, and $\left.g_{S}\right)$, and the average momentum fraction $\langle x\rangle_{u-d}$.

Results are shown in the "plateau plots" of Fig. 6. The behavior depends strongly on the observable. For $g_{A}, \chi_{\text {opt }}$ produces a much stronger dependence on the operator insertion time, $\tau$, indicating a significant enhancement of excited-state contributions. The opposite is true for $g_{T}$, where excited-state effects appear to be significantly suppressed when using $\chi_{\text {opt }}$. The difference between the two operators is relatively small for $g_{S}$ and $\langle x\rangle_{u-d}$. It is particularly problematic that $\chi_{\mathrm{opt}}$, which appears to be an improved operator based on the two-point correlator, produces significantly enhanced excited-state effects in $g_{A}$. In addition, across all observables $\chi_{\text {opt }}$ produces consistently larger statistical uncertainties.

We have also explored the use of simultaneous four-state fits to two-point and three-point correlators. However, for
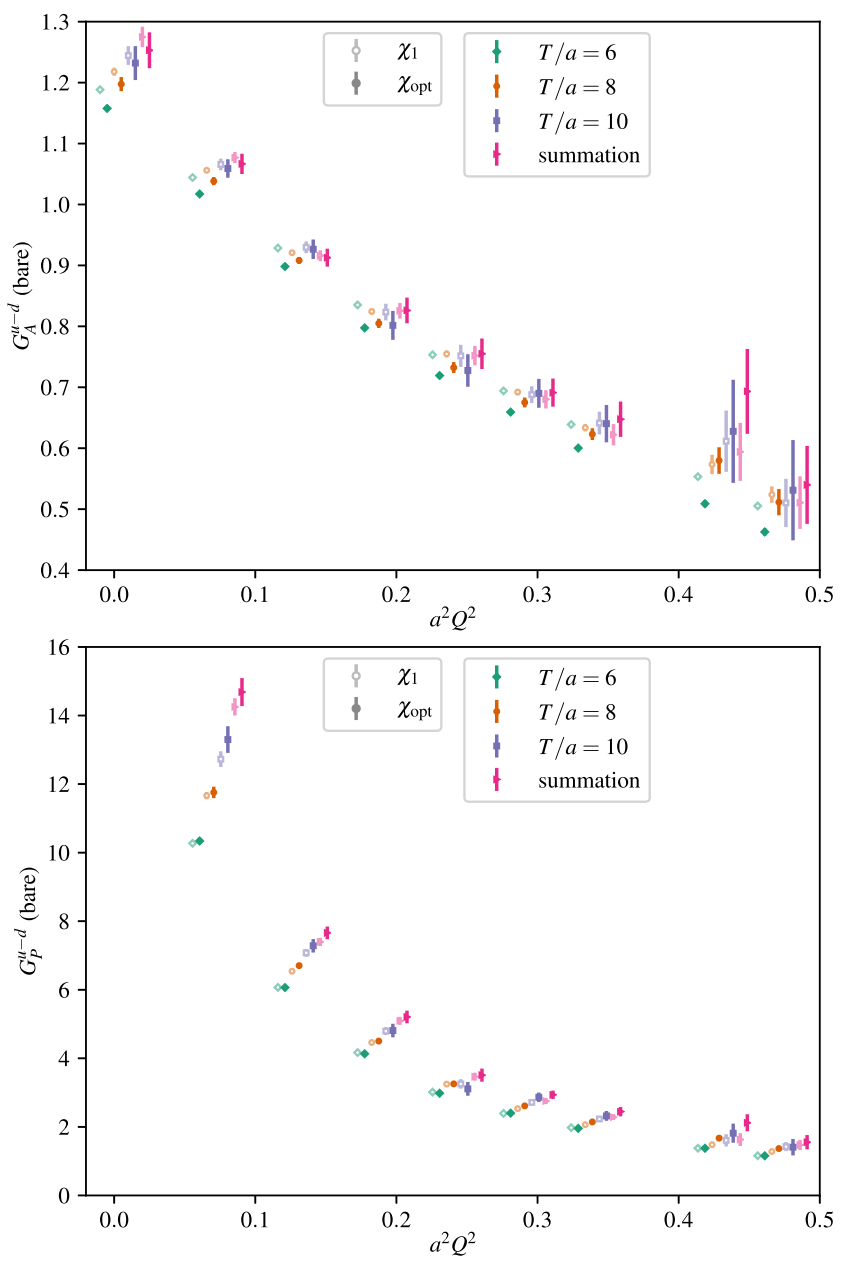

FIG. 8. Isovector axial and induced pseudoscalar form factors, $G_{A}$ and $G_{P}$. See the caption of Fig. 7.

each operator $\mathcal{O}$ the corresponding fit model has ten independent unknown matrix elements $\left\langle n^{\prime}|\mathcal{O}| n\right\rangle$. Since we have computed three-point correlators with only two combinations of source and sink interpolators, the fits are unable to constrain most of the operator matrix elements. This prevents the use of four-state fits to understand what causes the differences between $\chi_{1}$ and $\chi_{\mathrm{opt}}$ for estimating matrix elements.

\section{Form factors}

The isovector form factors $F_{1}$ and $F_{2}$ of the electromagnetic current are shown in Fig. 7 and the form factors $G_{A}$ and $G_{P}$ of the axial current are shown in Fig. 8. The results are rather mixed. For $F_{2}$ near $a^{2} Q^{2}=0.3$ and for $F_{1}, \chi_{\text {opt }}$ produces a weaker dependence on the source-sink separation than $\chi_{1}$, indicating the suppression of excitedstate contributions. However, the opposite is true for $F_{2}$ at lower $Q^{2}$ and for $G_{A}$. For $G_{P}$, the two operators produce similar excited-state effects, which are very large at low $Q^{2}$. Chiral perturbation theory predicts this large excited-state contribution [25] as a result of nucleon-pion states, which the hybrid operators are unlikely to help remove. 


\section{CONCLUSIONS}

The use of a variational basis comprising a standard interpolating operator and hybrid ones presents the possibility of reducing excited-state contamination in nucleon structure calculations at low computational cost. In twopoint correlators, this is borne out, as seen in Figs. 1 and 5. However, in nucleon structure observables that depend on three-point correlators there is no consistent result. The tensor charge shows significantly reduced excited-state effects, whereas the axial charge shows increased effects. ${ }^{5}$ Other observables show little change and for form factors the result can depend on $Q^{2}$. If one also takes into account the increased statistical uncertainty, then the setup using hybrid interpolators appears to be not worth pursuing further in its current form.

Results from the four-state fit indicate that the variational procedure succeeds at suppressing some excited states while another lower-lying state is unaffected. The presence of many relevant excited states suggests ways of understanding the results: one possibility is that for the axial charge, the variational procedure spoils a partial cancellation of contributions between two different excited states. Since operator matrix elements do not play a role in the GEVP, there is no guarantee that reducing the net contribution from excited states in the two-point correlator will do the same in three-point correlators.

\footnotetext{
${ }^{5}$ The opposite effect was seen using a different basis of interpolators in Ref. [7], where the variational analyses produced reduced excited-state effects for $g_{A}$ and enhanced excited-state effects for $g_{T}$, compared with the interpolator $S_{5}$.
}

Reliably improved results using the variational method can only be obtained by being in the asymptotic regime of Ref. [4]. This means that all low-lying excitations must be identified, including multiparticle states, which in practice require nonlocal interpolators. Doing so amounts to a challenging computation; however, as this work has shown, half-measures (such as using a small number of local interpolators) do not consistently reduce excited-state contamination in nucleon structure observables.

\section{ACKNOWLEDGMENTS}

We thank the Budapest-Marseille-Wuppertal collaboration for making their configurations available to us. Calculations for this project were done using the Qlua software suite [35], and we made use of the QOPQDP adaptive multigrid solver [36,37]. This research used resources on the supercomputer JUWELS [38] at Jülich Supercomputing Centre (JSC); we acknowledge computing time granted by the John von Neumann Institute for Computing (NIC). S.M. is supported by the U.S. Department of Energy (DOE), Office of Science, Office of High Energy Physics under Award No. DE-SC0009913. S. M. and S. S. are also supported by the RIKEN BNL Research Center under its joint tenure track fellowships with the University of Arizona and Stony Brook University, respectively. M. E., J. N., and A. P. are supported in part by the Office of Nuclear Physics of the U.S. DOE under Grants No. DE-FG02-96ER40965, No. DE-SC-0011090, and No. DE-SC0018121, respectively. S. K. and N.H. received support from Deutsche Forschungsgemeinschaft Grant No. SFB-TRR 55.
[1] G. P. Lepage, The analysis of algorithms for lattice field theory, in From Actions to Answers: Proceedings of the 1989 Theoretical Advanced Study Institute in Elementary Particle Physics, edited by T. DeGrand and D. Toussaint (World Scientific, Singapore, 1989), pp. 97-120.

[2] C. Michael, Adjoint sources in lattice gauge theory, Nucl. Phys. B259, 58 (1985).

[3] M. Lüscher and U. Wolff, How to calculate the elastic scattering matrix in two-dimensional quantum field theories by numerical simulation, Nucl. Phys. B339, 222 (1990).

[4] B. Blossier, M. Della Morte, G. von Hippel, T. Mendes, and R. Sommer, On the generalized eigenvalue method for energies and matrix elements in lattice field theory, J. High Energy Phys. 04 (2009) 094.

[5] G. Engel, C. Gattringer, L. Ya. Glozman, C. B. Lang, M. Limmer, D. Mohler, and A. Schäfer, Baryon axial charges from chirally improved fermions: First results, Proc. Sci., LAT2009 (2009) 135 [arXiv:0910.4190].
[6] B. J. Owen, J. Dragos, W. Kamleh, D. B. Leinweber, M. S. Mahbub, B. J. Menadue, and J. M. Zanotti, Variational approach to the calculation of $g_{A}$, Phys. Lett. B 723, 217 (2013).

[7] B. Yoon et al., Controlling excited-state contamination in nucleon matrix elements, Phys. Rev. D 93, 114506 (2016).

[8] J. Dragos, R. Horsley, W. Kamleh, D. B. Leinweber, Y. Nakamura, P. E. L. Rakow, G. Schierholz, R. D. Young, and J. M. Zanotti, Nucleon matrix elements using the variational method in lattice QCD, Phys. Rev. D 94, 074505 (2016).

[9] F. M. Stokes, W. Kamleh, and D. B. Leinweber, Oppositeparity contaminations in lattice nucleon form factors, Phys. Rev. D 99, 074506 (2019).

[10] J. Green, M. Engelhardt, S. Krieg, S. Meinel, J. Negele, A. Pochinsky, and S. Syritsyn, Nucleon form factors with light Wilson quarks, Proc. Sci., LATTICE2013 (2014) 276 [arXiv:1310.7043].

[11] J. R. Green, J. W. Negele, A. V. Pochinsky, S. N. Syritsyn, M. Engelhardt, and S. Krieg, Nucleon electromagnetic form 
factors from lattice QCD using a nearly physical pion mass, Phys. Rev. D 90, 074507 (2014).

[12] K. Ottnad, T. Harris, H. Meyer, G. von Hippel, J. Wilhelm, and $\mathrm{H}$. Wittig, Nucleon average quark momentum fraction with $N_{\mathrm{f}}=2+1$ Wilson fermions, EPJ Web Conf. 175, 06026 (2018).

[13] C. Aubin and K. Orginos, A new approach for Delta form factors, AIP Conf. Proc. 1374, 621 (2011).

[14] C. Aubin and K. Orginos, An improved method for extracting matrix elements from lattice three-point functions, Proc. Sci., LATTICE2011 (2011) 148.

[15] C. Egerer, D. Richards, and F. Winter, Controlling excitedstate contributions with distillation in lattice QCD calculations of nucleon isovector charges $g_{S}^{u-d}, g_{A}^{u-d}, g_{T}^{u-d}$, Phys. Rev. D 99, 034506 (2019).

[16] J. J. Dudek and R. G. Edwards, Hybrid baryons in QCD, Phys. Rev. D 85, 054016 (2012).

[17] C. B. Lang, L. Leskovec, M. Padmanath, and S. Prelovsek, Pion-nucleon scattering in the Roper channel from lattice QCD, Phys. Rev. D 95, 014510 (2017).

[18] S. Dürr, Z. Fodor, C. Hoelbling, S. D. Katz, S. Krieg, T. Kurth, L. Lellouch, T. Lippert, K. K. Szabó, and G. Vulvert, Lattice QCD at the physical point: Simulation and analysis details, J. High Energy Phys. 08 (2011) 148.

[19] B.C. Tiburzi, Time dependence of nucleon correlation functions in chiral perturbation theory, Phys. Rev. D 80, 014002 (2009).

[20] B. C. Tiburzi, Chiral corrections to nucleon two- and threepoint correlation functions, Phys. Rev. D 91, 094510 (2015).

[21] O. Bär, Nucleon-pion-state contribution to nucleon twopoint correlation functions, Phys. Rev. D 92, 074504 (2015).

[22] O. Bär, Nucleon-pion-state contribution in lattice calculations of the nucleon charges $g_{A}, g_{T}$ and $g_{S}$, Phys. Rev. D 94, 054505 (2016).

[23] M. T. Hansen and H. B. Meyer, On the effect of excited states in lattice calculations of the nucleon axial charge, Nucl. Phys. B923, 558 (2017).

[24] O. Bär, Chiral perturbation theory and nucleon-pion-state contaminations in lattice QCD, Int. J. Mod. Phys. A 32, 1730011 (2017).

[25] O. Bär, $N \pi$-state contamination in lattice calculations of the nucleon axial form factors, Phys. Rev. D 99, 054506 (2019).
[26] J. Green, Systematics in nucleon matrix element calculations, Proc. Sci., LATTICE2018 (2018) 016 [arXiv:1812 .10574].

[27] J. J. Dudek, R. G. Edwards, and C. E. Thomas (Hadron Spectrum Collaboration), Energy dependence of the $\rho$ resonance in $\pi \pi$ elastic scattering from lattice QCD, Phys. Rev. D 87, 034505 (2013); Phys. Rev. D 90, 099902(E) (2014).

[28] M. Göckeler, R. Horsley, E.-M. Ilgenfritz, H. Perlt, P. E. L. Rakow, G. Schierholz, and A. Schiller, Polarized and unpolarized nucleon structure functions from lattice QCD, Phys. Rev. D 53, 2317 (1996).

[29] S. Basak, R. Edwards, G. T. Fleming, U. M. Heller, C. Morningstar, D. Richards, I. Sato, and S. J. Wallace, Clebsch-Gordan construction of lattice interpolating fields for excited baryons, Phys. Rev. D 72, 074501 (2005).

[30] B. Sheikholeslami and R. Wohlert, Improved continuum limit lattice action for QCD with Wilson fermions, Nucl. Phys. B259, 572 (1985).

[31] S. Güsken, A study of smearing techniques for hadron correlation functions, Nucl. Phys. B, Proc. Suppl. 17, 361 (1990).

[32] J. R. Green, J. W. Negele, A. V. Pochinsky, S. N. Syritsyn, M. Engelhardt, and S. Krieg, Nucleon scalar and tensor charges from lattice QCD with light Wilson quarks, Phys. Rev. D 86, 114509 (2012).

[33] J. R. Green, M. Engelhardt, S. Krieg, J. W. Negele, A. V. Pochinsky, and S. N. Syritsyn, Nucleon structure from lattice QCD using a nearly physical pion mass, Phys. Lett. B 734, 290 (2014).

[34] C. Morningstar and M. J. Peardon, Analytic smearing of $S U$ (3) link variables in lattice QCD, Phys. Rev. D 69, 054501 (2004).

[35] A. Pochinsky, Qlua, https://usqcd.lns.mit.edu/qlua.

[36] R. Babich, J. Brannick, R. C. Brower, M. A. Clark, T. A. Manteuffel, S. F. McCormick, J. C. Osborn, and C. Rebbi, Adaptive Multigrid Algorithm for the Lattice Wilson-Dirac Operator, Phys. Rev. Lett. 105, 201602 (2010).

[37] J. Osborn et al., QOPQDP, https://usqcd-software.github.io/ qopqdp/.

[38] Jülich Supercomputing Centre, JUWELS: Modular Tier-0/1 supercomputer at the Jülich supercomputing centre, J. Large-Scale Res. Facil. 5, A135 (2019). 